\title{
¿Qué esperan los Docentes de los Agentes Conversacionales Pedagógicos?
}

\section{What School Teachers Expect from Conversational Pedagogic Agents?}

Silvia Tamayo, Diana Pérez-Marín

Departamento de Ciencias de la Computación, Arquitectura de Computadores, Lenguajes y Sistemas Informáticos, Estadística e Investigación Operativa, Escuela Técnica Superior de Ingeniería Informática, Universidad Rey Juan Carlos

Calle Tulipán s/n, 28933 Móstoles, Madrid, España

diana.perez@urjc.es (http://orcid.org/0000-0003-3390-0251),silviatamayomoreno@gmail.com

\section{Resumen}

Los Agentes Conversacionales Pedagógicos son sistemas interactivos que enseñan a los estudiantes asumiendo el rol de profesor, estudiante - acompañante mediante un diálogo en lenguaje natural. Mucho se ha investigado sobre los dominios en los que se pueden usar los agentes, los resultados a nivel de eficacia educativa y nivel de satisfacción y motivación de los estudiantes. Sin embargo, en la literatura no se encuentran muchos ejemplos de estudios que revelen la opinión de los docentes sobre este tipo de tecnología educativa. En este artículo, se proporcionan los resultados de una encuesta realizada a 82 docentes de 4 colegios de la zona sur de Madrid para saber qué esperan de los agentes. En particular, se dará respuesta a las siguientes preguntas de investigación: P1) iconocen esta tecnología educativa?, P2) Si es así, ¿están acostumbrados al uso de este tipo de tecnologías en el aula? y P3) Si tuvieran poder de decisión en el diseño del agente, ¿qué características consideran que son las más adecuadas? ¿cómo debería reaccionar el agente antes diversas situaciones? De los resultados obtenidos, se espera proporcionar información a todos los investigadores, diseñadores y profesores que quieran integrar este tipo de tecnología educativa en su aula.

\section{Palabras Clave}

Agente Conversacional Pedagógico; Educación Infantil; Educación Primaria; Diseño de Tecnología Educativa

\section{Abstract}

Conversational Pedagogical Agents are interactive systems that teach students by assuming the role of teacher, student or companion through a natural language dialogue. If has been investigated a lot on the domains in which the agents can be used, the results in terms of educational effectiveness and level of satisfaction and motivation of the students. However, in the literature, there are not many examples of studies that reveal the opinion of school teachers on this type of educational technology. In this paper, we provide the results of a survey of 82 teachers to know what they expect from the agents. The following research questions will be answered: Q1) Do you know this educational technology? Q2) If so, are teachers used to integrate this type of technology in the classroom? and, P3) If teachers could design the agent, what characteristics do they consider to be the most appropriate? How should the agent react to different situations? From the results gathered, it is expected to provide information to all researchers, designers and teachers who want to integrate this type of educational technology in their classroom.
Keywords

Conversational Pedagogical Agent; Pre-Primary Education; Primary Education; Educational Technology Design 


\section{Introducción}

Los Agentes Conversacionales Pedagógicos (ACP) son sistemas interactivos educativos que permiten a los estudiantes repasar de una forma entretenida y amigable mediante un diálogo con el ordenador (Johnson, et al., 2000).

En la actualidad, existen cientos de agentes distintos (Pérez-Marín y Pascual-Nieto, 2011) aplicados en dominios muy diversos, desde la enseñanza de Sistemas Operativos en la Universidad (Graesser, et al., 2001), Ciencias Naturales en Educación Primaria, o competencias como la capacidad de contar cuentos (Ryokai, et al., 2003) o empatizar con otras culturas en Educación Primaria (Hays, et al., 2009).

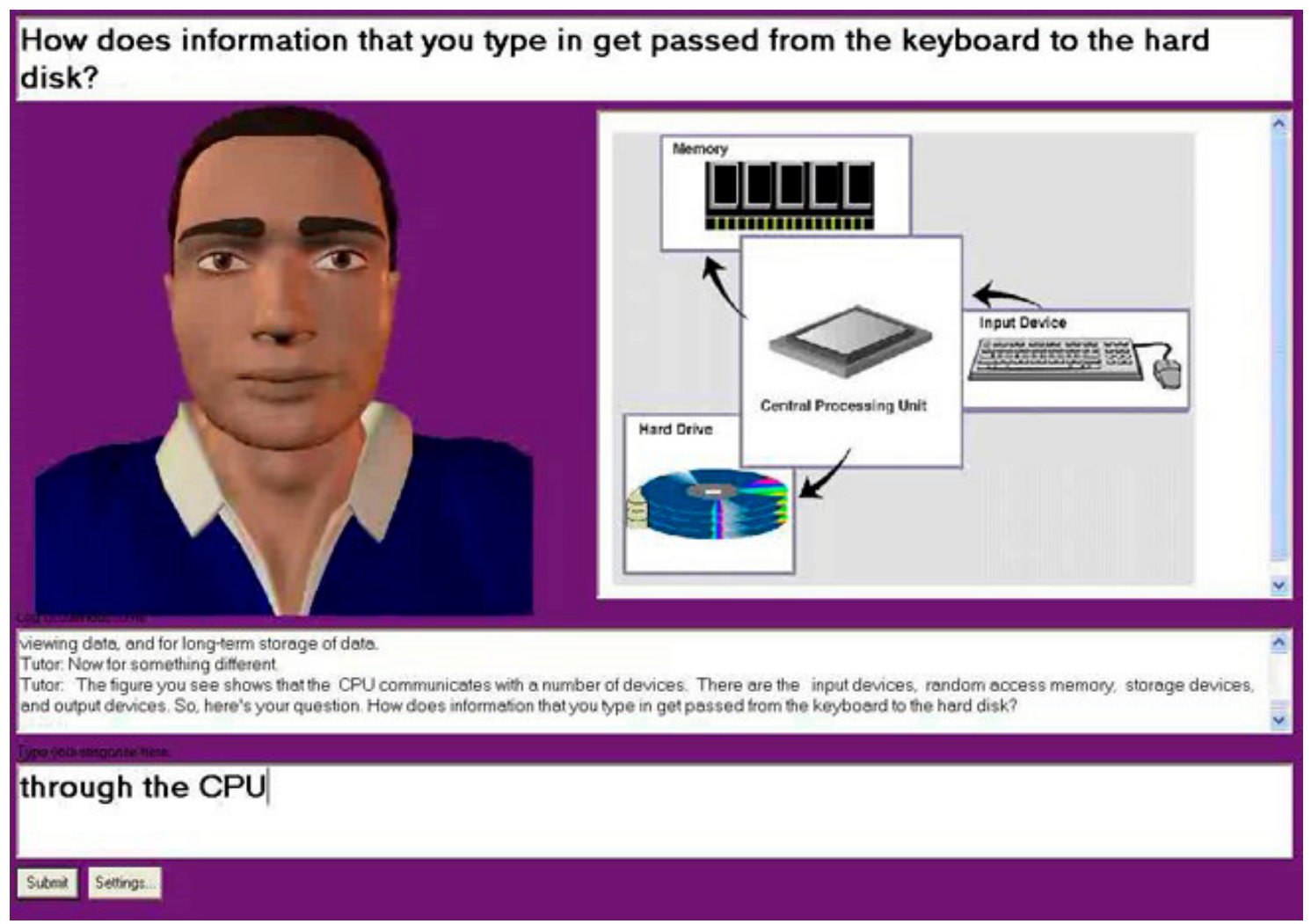

Figura 1. AutoTutor (Person y Graesser, 2000)

Los resultados conseguidos en niveles universitarios son prometedores, con mejoras de hasta 0.8 en la puntuación final del examen (escala 0-10) de los estudiantes que usaron AutoTutor (Graesser, et al., 2001), según los experimentos realizados por sus creadores. Puede verse una imagen del mismo en la Figura 1.

En el caso de los niveles inferiores, algunos autores como Schroeder et al. (2013) han reportado en sus estudios resultados como que el efecto de aprender con agentes pedagógicos, aunque no es muy elevado, es estadísticamente significativo en comparación con sistemas que no usan agentes. Además, Schroeder et al. (2013) indican que los agentes pedagógicos proporcionan beneficios en la 
educación hasta 12 años, especialmente entre los 9 y 12 años. Aunque añaden que los resultados deben interpretarse con cautela debido al bajo número de estudios existentes en estas edades.

Otros autores reportan beneficios como el efecto Persona (Lester, et al., 1997), según el cual la mera presencia de una cara en la interfaz supone un efecto positivo en el aprendizaje con el agente, el efecto Proteo (Yee y Bailenson, 2007) según el cual los estudiantes quieren parecerse al agente y esto puede ser una fuente de motivación para aprender, y el efecto Protégé (Chase, et al., 2009), según el cual cuando un estudiante se convierte en profesor del agente, adquiere una responsabilidad, que le puede motivar a aprender más para poder enseñar correctamente al agente.

En la revisión de la literatura de agentes, la mayoría de los artículos se centran en la descripción del agente, los resultados obtenidos a nivel de eficacia educativa, o satisfacción de los estudiantes, pero no se reporta o se reporta muy brevemente la opinión de los profesores. Además, se reportan casos de uso de Universidad, Educación Secundaria y Primaria, sin encontrar ejemplos de uso de los ACP en Educación Infantil (Tamayo-Moreno, 2017).

Por este motivo, este trabajo se centra en los docentes y su relación con los agentes. En particular, se va a intentar dar respuesta a las siguientes cuestiones:

P1) ¿Conocen los profesores esta tecnología educativa?

P2) Si es así, ¿están acostumbrados al uso de este tipo de tecnologías en el aula?

P3) Si tuvieran poder de decisión en el diseño del agente, ¿qué características consideran que son las más adecuadas? ¿Cómo debería reaccionar el agente antes diversas situaciones?

De los resultados obtenidos, se espera proporcionar información a todos los investigadores, diseñadores y profesores que quieran integrar este tipo de tecnología educativa en su aula.

Para ello, se ha realizado una encuesta a 82 docentes (52,4\% hombres; $47,6 \%$ mujeres). Las edades de los encuestados arrojan un perfil bastante joven, situándose la media de edad en 27 años. El contexto en el que se ha llevado a cabo esta investigación ha sido en cuatro colegios de la zona sur de la Comunidad de Madrid, tanto públicos como privados, en las áreas de Educación Infantil y Primaria. El periodo de realización abarcó desde el 16 de abril de 2015 hasta el 23 de julio de 2015. De los resultados obtenidos se ha podido contestar a las tres preguntas planteadas.

El artículo se estructura en cinco apartados: el segundo apartado revisa la literatura de los ACP; el tercer apartado presenta la encuesta realizada y el proceso de recogida de los resultados; el cuarto apartado recoge los resultados obtenidos y su análisis; y, finalmente, el quinto apartado termina con las respuestas a las preguntas de investigación planteadas. 


\section{Estado del arte}

\subsection{Definición}

Los agentes conversacionales se definen como guías, docentes, ayudantes, la personalidad de la máquina o de un programa específico (Domínguez, 2011). En interfaces conversacionales, el uso que se le da es la búsqueda de una interacción más personalizada entre la máquina y el usuario. Algunas de las características de los agentes son: autonomía, proactividad, sociabilidad, tienen que tener una personalidad, reactividad y persistencia (Mas, 2005).

Los agentes animados o conversacionales son entidades que se pueden representar con personas, animales, cosas que pueden hablar con sonido o texto (Tatar, et al., 2013; Clark, et al., 2013). Los agentes son un apoyo para una amplia gama de aplicaciones en empresas comerciales, salud, entrenamiento, educación (Kuz y Falco, 2015).

Debido a la importancia que está adquiriendo la tecnología en la educación, la posibilidad de integrar agentes en entornos de aprendizaje también está aumentando y ganando en importancia (Veletsianos, et al., 2009). En este sentido, son importantes los Agentes Conversacionales Pedagógicos (ACP), que se pueden definir como sistemas interactivos que permiten a los estudiantes repasar de una forma entretenida y amigable (Johnson, et al., 2000). Puede verse un ejemplo del ACP AutoTutor en la Figura 1.

A medida que aumenta el uso de los ACP, se hace especialmente relevante la compresión de cómo diseñar estos personajes para la enseñanza y el aprendizaje, fomentando la interacción entre humanos y agentes, algo que supone un desafío y que hasta el momento no se ha alcanzado completamente (Van Vuuren, 2007). Sirva como ejemplo alguno de los problemas, aún no resueltos, que conlleva la interacción con agentes o la incomprensión del diálogo. Esto genera sentimientos negativos en el estudiante, dificulta la comunicación e interacción y, en último lugar, disminuye la consecución de las tareas de aprendizaje (Veletsianos, et al., 2009). En ello radica la importancia del diseño, así como de todos los avances que se pueda alcanzar en ese sentido, tanto en la estandarización como el establecimiento de metodologías de diseño.

\subsection{Taxonomías}

Los ACP se usan en los entornos de aprendizaje con diferentes objetivos como facilitar el aprendizaje y motivar (Khosrow-Pour, 2008). 
Nanne (2015) hace una recopilación de los principales criterios de categorización de los agentes pedagógicos. Dichos criterios son: el carácter del agente, el tipo de entorno en que está integrado el agente (los principales son entornos de aprendizaje virtual y sistemas tutores inteligentes), el papel educativo que el agente desempeña en este entorno (diferentes estudios de investigación se centran en los roles, así Ryu y Baylor (2005) y Baylor y Ebbers (2003) los clasifican en experto mentor y motivador, o Smith et al. (1999) en facilitador, mentor y asesor), los componentes no verbales que tiene el agente (mirada, gesto, expresiones faciales, emociones), la multiplicidad de agentes en una aplicación (sistemas con un único agente educativo o con múltiples), la gestión del aprendizaje colaborativo, áreas de aplicación y condiciones en la entrada que tenga en cuenta el agente pedagógico.

También se pueden distinguir según su interfaz gráfica, en agentes animados con cuerpo capaces de interactuar con comunicación verbal y no verbal, que son los ECA's (Embodied Conversational Agents) o Agentes Conversacionales Corpóreos o Animados, y aquellos agentes que son solo un rostro o un dibujo estático. Pérez-Marín (2011) recoge diez criterios principales para clasificar los agentes según su rol, capacidad de interacción, tipo de animación, posibilidades afectivas, personaje virtual, capacidad de evolución-adaptación, ubicuidad, dominio y edad del estudiante.

\subsection{Revisión de agentes}

Según los criterios revisados en la Sección 2.2, en este apartado se seleccionan tres agentes según su rol de profesor, estudiante o acompañante, con el objetivo de proporcionar una visión global de las posibilidades de los agentes. Para todos los agentes revisados se seguirá la misma estructura: descripción global, metodología, algoritmo, uso y resultados. Una revisión más completa de los agentes puede encontrarse en Tamayo-Moreno (2017).

\subsubsection{Agente con rol de profesor: AutoTutor}

Es un sistema de tutoría inteligente que hace uso de un agente conversacional animado con expresiones faciales, habla sintetizada y gestos. Se basa en teorías constructivistas, teniendo iniciativa mixta de diálogo y animación 3D (Graesser, et al., 2001), siendo un referente desde los años 90 (Person y Graesser, 2000). Ha sido desarrollado por investigadores del Instituto para Sistemas Inteligentes de la Universidad de Memphis. Puede verse una imagen de AutoTutor en la Figura 1.

Metodología. No se describe. Fue diseñado por un equipo interdisciplinario de la investigación que abarca la informática, la ingeniería, la psicología, la ciencia cognitiva, la lingüística, la física, y la educación (D’mello y Graesser, 2012).

Características en las que se basa el Algoritmo. La arquitectura de AutoTutor se compone de cinco 
módulos principales: un agente animado, una secuencia de comandos del currículum, analizadores de lenguaje, análisis semántico latente (LSA) y un generador de movimiento de diálogo que se centra en el diálogo en lenguaje natural. AutoTutor utiliza algoritmos de lingüística computacional incluyendo correspondencia de expresiones regulares y clasificadoras de actos de habla. El diálogo del AutoTutor se adapta al nivel del conocimiento del usuario, incluida la conversación, reaccionando de forma pedagógicamente adecuada. Fueron diseñados para modelar estilos pedagógicos, patrones de diálogo, lenguaje y los gestos de tutores humanos.

Uso-Integración. AutoTutor ha demostrado ganancias de aprendizaje en más de una docena de experimentos con estudiantes universitarios para los temas de introducción a los conocimientos informáticos y la física newtoniana. Ha sido probado en más de mil estudiantes (VanLehn, et al., 2007). Ha contribuido a la ciencia de cómo la gente aprende, así como a soluciones de ingeniería para aumentar el aprendizaje (D’mello y Graesser, 2012).

Evaluación de resultados. Según sus creadores es capaz de aumentar hasta 0.8 puntos en el resultado final de los exámenes (D’mello y Graesser, 2012).

Se han hecho estudios contrastando la efectividad de mostrar el diálogo únicamente, sin y con la presencia del agente, comprobándose que cuando el agente está presente los resultados son mejores, lo que se conoce como efecto Persona. Algunos estudios indican que parte de su eficacia puede deberse al contenido del diálogo que va siendo presentado.

\subsubsection{Agente con rol de estudiante: Betty}

Es un agente al que los estudiantes enseñan utilizando una representación visual bien estructurada (Leelawong y Biswas, 2008). Utilizando el desempeño de su agente (en función de cómo se le enseña) como una motivación, los estudiantes aprenden para intentar mejorar el conocimiento del agente y, en este proceso, aprenden mejor por sí mismos. Se utiliza para la enseñanza de la asignatura de ciencias en Primaria. Puede verse una imagen de Betty y Mr. Davis en la Figura 2.

Metodología. No se describe.

Características en las que se basa el Algoritmo. El estudiante enseña al agente mediante mapas de conceptos, de tal manera que, en función de estos, responderá de forma razonada a las preguntas del alumno. Como las respuestas pueden ser correctas o no, para supervisarlo, existe otro agente Mr. Davis, que tiene el rol de tutor. Este pude interactuar con el estudiante, animándole para que haga preguntas a Betty si hace tiempo que no lo hace. Y si el estudiante no sabe relacionar conceptos, se encarga de explicarle. 


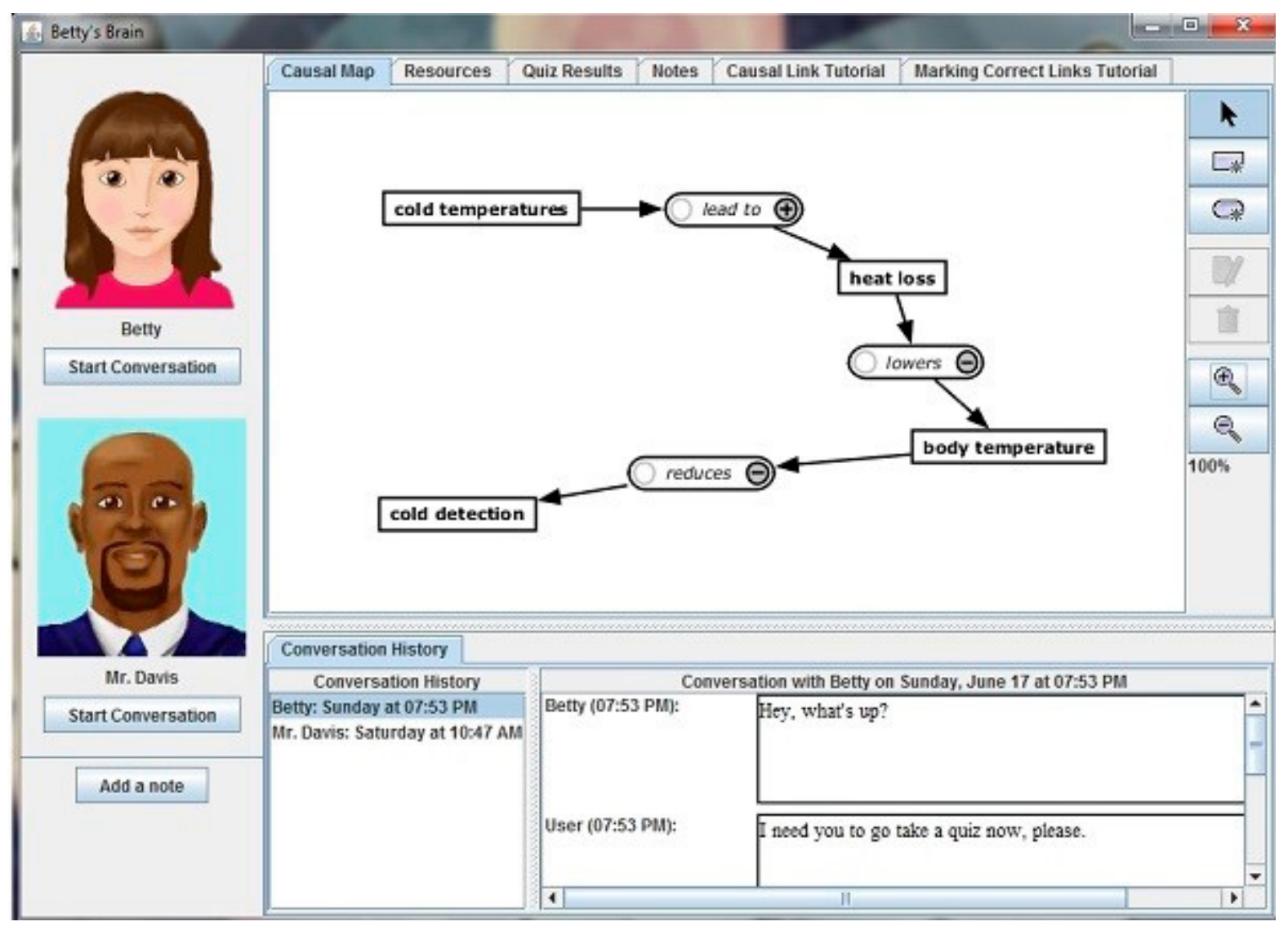

Figura 2. Interfaz del agente Betty (Leelawong y Biswas, 2008)

Uso-Integración. Betty se ha probado con estudiantes de colegio en el área de Ciencias Naturales. Los participantes del estudio fueron 56 estudiantes de dos aulas de ciencias de quinto grado, impartidas por el mismo profesor.

Evaluación de resultados. Los resultados obtenidos prueban que es efectivo en la ayuda a los estudiantes en las preguntas de respuesta libre, a responder mejor (Biswas, et al., 2009). El estudio, junto con la investigación previa, parece demostrar que trabajar con Betty es útil porque apoya el compromiso de los estudiantes y promueve procesos cognitivos en la educación (Biswas, et al., 2009).

\subsubsection{Agentes con rol de acompañante: SBEL}

Es un acompañante del estudiante para aprender portugués brasileño en el entorno de e-learning basado en escenarios (Reategui, et al., 2007), con situaciones que se encontrarían en la vida real, lo que permite un aprendizaje más natural. Puede verse una imagen del agente en la Figura 3.

Metodología. No se describe.

Características en las que se basa el Algoritmo. Según Paulus et al. (2006) un aprendizaje debe proporcionarse en un contexto en el que se ofrezcan consejos útiles o modelos que ayuden a los estudiantes a lograr sus objetivos de resolver tareas. Los entornos de e-learning basados en escenarios (SBeL) pueden encajar en este modelo, puesto que suelen presentar una historia (video, animación o 
audio) a ser seguida por los estudiantes, para percibir acciones y actitudes en la vida real, que servirá de base en la realización de tareas y discusiones posteriores. De esta forma SBeL también puede ofrecer un entorno interactivo con tareas auténticas y significativas distribuidas de forma no lineal, donde los estudiantes pueden centrarse en actividades contextualizadas (Reategui, et al., 2007).

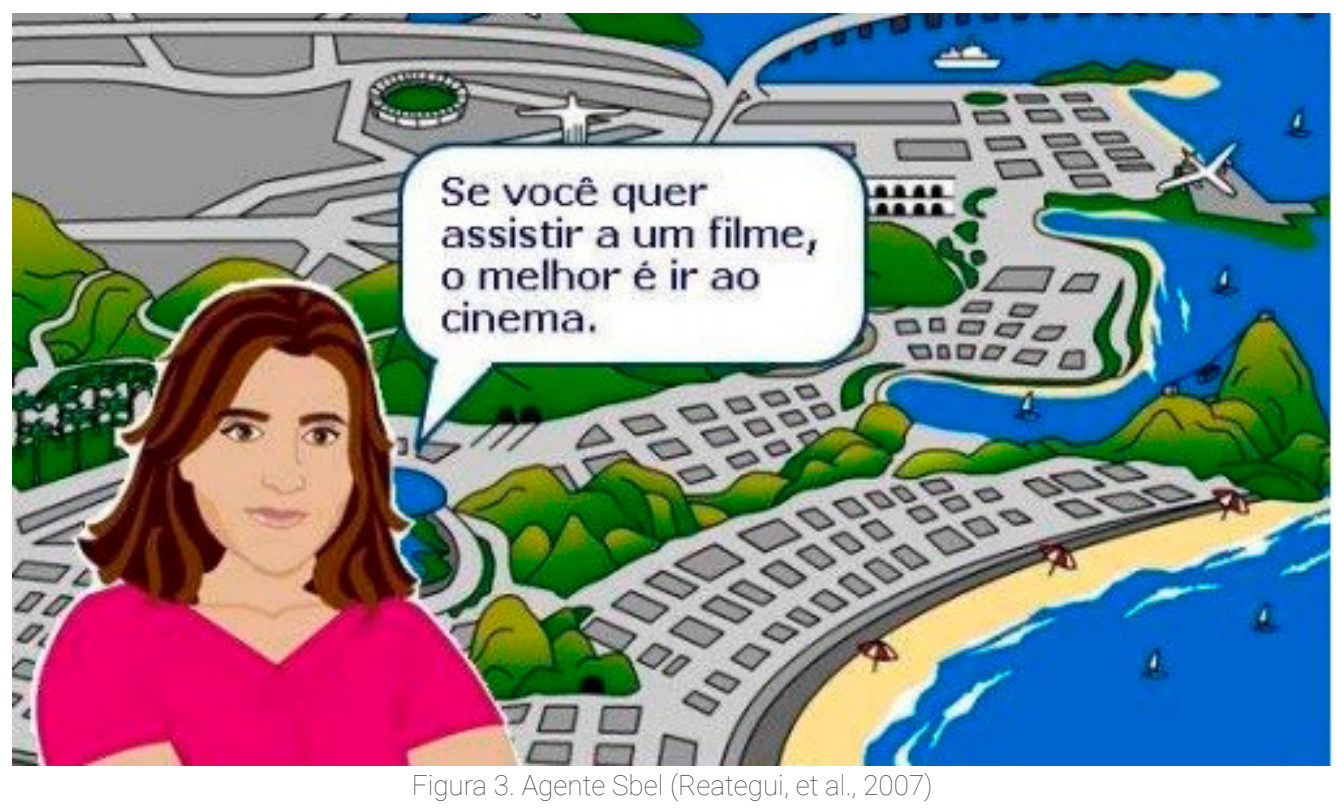

Utiliza un avatar que acompaña al usuario en diferentes situaciones reales empleando el vocabulario y las expresiones indicadas en cada momento. Los estudiantes, aprenden el vocabulario en situaciones que se encontrarían en la vida real, lo que permite un aprendizaje más natural (Reategui, et al., 2007).

Uso-Integración. Se llevó a cabo un caso de estudio para aprender portugués brasileño. Los estudiantes matriculados en el curso participan en un juego de roles en línea, en el que actúan como participantes en un programa de intercambio, viviendo con una familia brasileña en la ciudad de Río de Janeiro.

El enfoque educativo adaptado combina el aprendizaje basado en tareas y escenarios (Reategui, et al., 2007).

Kim y Baylor (2006) recomiendan el comportamiento proactivo de los agentes en el proceso de aprendizaje, ya que los estudios demuestran que los ayudantes integrados raramente se utilizan.

Evaluación de resultados. Los materiales de aprendizaje se diseñan para proveer tareas reales de aprendizaje contextual y ofrecen diferentes caminos exitosos, como lo sugiere Kindley (2002a, 2002b). La comunicación intercultural y la conciencia cultural serán fomentadas por discusiones adicionales, como sugirió Ziegahn (2001). En relación al uso de agentes, los personajes se están desarrollando siguiendo un modelo de coherencia global, que incluye rasgos como la conversación, la empatía, la sociabilidad, la inteligencia y la variabilidad, tal como fue destacado por Reategui y Moraes (2006). 


\section{Encuesta}

\subsection{Creación de la encuesta}

La encuesta está formada por 24 preguntas para docentes de diferentes tipos:

- Tipo test: selección de respuesta entre varias alternativas, se intenta obtener información objetiva.

- $\quad$ Tipo texto: se trabaja con preguntas simples y elaboradas. Las primeras, aportan aspectos personales de cada encuestado. Las segundas, tratan de capturar información acerca de impresiones, ideas y opiniones personales sobre el agente conversacional, siendo de utilidad para analizarlas y valorarlas con objeto de identificar posibles modificaciones que se traduzcan en mejoras.

- $\quad$ Tipo escala: preguntas de valoración, la escala es de 0 a 10, de menor a mayor importancia. Tratan de conocer la importancia que otorgan a determinados aspectos del agente para que sea motivador para el alumno.

Con objeto de facilitar la obtención de información significativa, se han considerado una serie de recomendaciones para la elaboración de la encuesta:

- Uso de vocabulario claro y sencillo en las preguntas, evitando construcciones ambiguas.

- Preguntas cortas que eviten aburrir al encuestado, aportando mayor dinamismo a la encuesta y motivando al estudiante a seguir rellenándola.

- Añadir un porcentaje mayor de preguntas cerradas que abiertas en la encuesta, ya que los encuestados suelen preferir preguntas cerradas, puesto que solo tienen que seleccionar la alternativa que recoja mejor sus preferencias.

Se considera relevante agradecer a los encuestados por su colaboración tanto al inicio como al final de la encuesta, ya que con ello hacen posible la investigación.

Las primeras nueve preguntas tienen como objetivo la investigación del uso de las tecnologías que hacen docentes, tanto en el aula como en casa, centrando la atención en su predisposición en el uso de la mismas, y si algunos aspectos personales, como género y edad, ponen de manifiesto diferencias significativas en su uso. 
La primera pregunta, tiene como objetivo conocer el género del encuestado para saber si está igualado o hay diferencia entre géneros.

La segunda pregunta pide el nombre del encuestado, lo que podría generar controversia, puesto que el encuestado podría no responder sinceramente ante la falta de anonimidad. Ante esta situación, se considera relevante tratar de transmitir seguridad e informarles de que sus datos personales no serán publicados, así como informar de que se debe a que en la fase de exploración es importante la indagación en los resultados obtenidos, y podría considerarse necesario hablar con ese profesor respecto a algunas de sus respuestas con objeto de profundizar en las mismas.

La tercera pregunta hace referencia a la edad del encuestado.

La cuarta pregunta pretende conocer el interés de los encuestados por las nuevas tecnologías, traduciéndose la pregunta a si los ordenadores les gustan o no, así como el motivo del uso: obligación, son conscientes de la utilidad de ello, les gusta emplearlo en su trabajo o por entretenimiento. Esta pregunta se complementa con la quinta, en la que se pregunta si tienen ordenador en casa.

En consonancia con las anteriores, se sitúa la sexta pregunta, que hace alusión a la disposición de Internet en casa. Igualmente relacionada está la séptima, que se interesa por la disposición de correo electrónico, y en caso de no disponer, si se desearía tenerlo. La octava pregunta cubre el uso del ordenador para buscar información y recursos para llevar al aula. La novena consulta sobre el uso del ordenador en su trabajo y, en caso afirmativo, si se usa en el aula, en casa o en ambas.

Los docentes poseen gran conocimiento de los estudiantes, por ello, se considera importante que valoren de 0 a 10 (siendo 0 el mínimo) la importancia que conceden a una serie de aspectos sobre las características que debería tener un programa de ordenador para motivar al alumno, por medio de nueve preguntas de tipo escala. Son, en orden incremental, las siguientes: que sea simpático o no, que el agente haga gestos con la cara y el cuerpo (siendo una ventaja o un inconveniente, por ejemplo una distracción para el alumno), que el agente conversacional aconseje a los alumnos durante la realización de las actividades propuestas, que hable en voz alta.

La decimoquinta se refiere a que anime a estudiar al alumno, considerando que muchos alumnos creen que estudiar no es una tarea entretenida, se intenta cambiar esta percepción por medio del uso del agente.

Las preguntas decimosexta y decimoséptima consultan acerca de que el agente indique al alumno que se ha equivocado y que pueda recordar sus opciones previas. Trata de identificar el punto en el que se han tenido dificultades, tener conocimiento de los pasos que le llevaron a ello, para poder superar la dificultad plateada. Esto supone un proceso de aprendizaje continuo. Por último, que tenga apariencia humana. 
La decimonovena pregunta pide opinión sobre si se considera que el uso del agente favorece la motivación de los alumnos. Las dos preguntas que le siguen platean situaciones que pueden darse durante el uso del mismo. Por un lado, la reacción del agente si el alumno no está atento, las alternativas se orientan en cómo volver a captar la atención del estudiante y mantenerla, y la motivación. Por otro lado, si el alumno no entiende algo, conocer la opinión basada en su experiencia educativa y metodológica ante situaciones similares.

La vigésimo segunda pregunta se centra en saber el uso semanal que a los encuestados les gustaría hacer uso del programa, así como el porqué de utilizarlo esas veces. Relacionada se encuentra la vigesimotercera, que plantea la idea de no acotar el uso del agente al aula, sino de que los estudiantes pudieran seguir usándolo en casa. Finalmente, se pregunta abiertamente a los encuestados, con objeto de que respondan con la mayor sinceridad posible, su opinión acerca de la propuesta de incorporar al aula un agente conversacional pedagógico.

\subsection{Proceso de recogida de datos}

El contexto en el que se ha llevado a cabo esta investigación ha sido en cuatro colegios de la zona sur de la Comunidad de Madrid, tanto públicos como privados, en las áreas de Educación Infantil y Primaria. La muestra de docentes está formada por los profesionales de la plantilla de cada centro que decidieron voluntariamente participar en los niveles seleccionados. Para rellenar la encuesta se les permitió emplear todo el tiempo que ellos estimasen oportuno, tanto en formato papel como de forma electrónica. Una vez entregada la encuesta ya no podía ser modificada.

Las encuestas de los docentes se realizaron principalmente en las aulas en las que se entregaron y en papel. La duración media de cada encuesta oscilaba entre 15 y 20 minutos. Cuando los docentes desconocían el uso del agente en el aula, hecho que le suscitaba duda en las respuestas a algunas de las preguntas, se les mostraba la imagen de los agentes mostrados en la Sección 2.3. para que pudieran hacerse una idea de cómo eran y cómo podría ser de utilidad para motivar a los alumnos en su aprendizaje.

Ningún profesor proporcionó un nombre de agente, en su lugar, los profesores que más entendían inicialmente el concepto preguntaban si era cómo los agentes que ayudan en tareas de redacción de documentos (ej. Clippy en Word), o ejercicios on-line para repasar inglés pero sin agente, solo como algo novedoso que querían comentar que usaban y preguntaban si era similar.

El hecho de poder realizar las encuestas en papel ofreció cierta flexibilidad a los docentes, puesto que algunos de ellos que tenían un tiempo limitado pudieron aprovechar instantes como el recreo, la 
entrada y la salida del colegio para realizarla. Además, algunos de ellos por falta de tiempo no podían realizar la encuesta en horario escolar, y ante esta situación, plantearon la idea de rellenarla en su casa y entregarla al día siguiente. En este caso, en el momento de entregar la encuesta a los docentes, se les preguntó si sabían lo que era un ACP, y en caso de que no lo supieran se les enseñaba para que la pudiesen rellenar posteriormente.

Como resumen se muestra la ficha técnica de la encuesta:

Técnica: cuestionario realizado en el centro en papel.

Ámbito geográfıco: Madrid.

Universo: profesores de colegios de Educación Infantil y Primaria.

Número de encuestas: 82.

Error: $+/-10,8 \%$ para un nivel de confianza del $95 \%$ y $p=q=0,5$.

Muestreo: selección aleatoria de colegios.

Trabajo de campo: desde el 16 de abril de 2015 hasta el 23 de julio de 2015.

\section{Resultados}

En este apartado se presentan los resultados de la encuesta realizada. El porcentaje de hombres encuestados fue del 52,4\% y el 47,6 restante de mujeres, como puede apreciarse en la Figura 4.

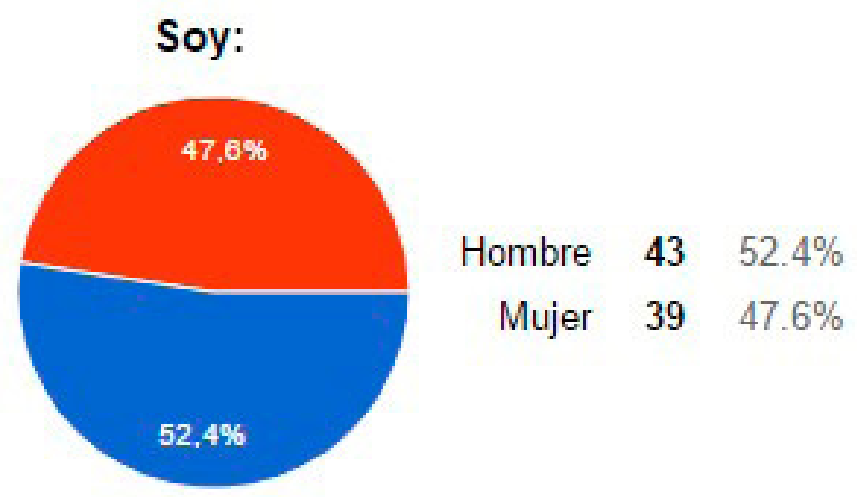

Figura 4. Sexo de los encuestados

Las edades de los encuestados, que pueden observase en la Figura 5 y en la Tabla 1, arrojan un perfil bastante joven, situándose la media de edad en casi 27 años. 


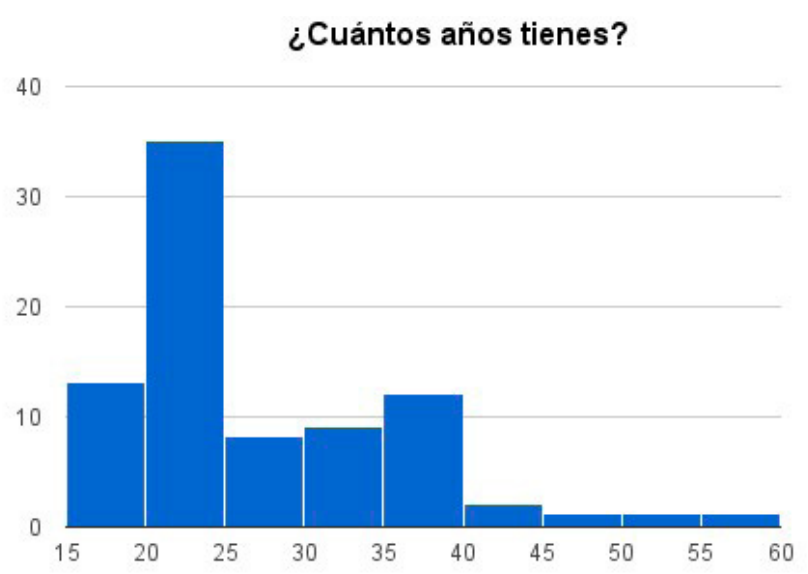

Figura 5. Años de los encuestados

\begin{tabular}{|c|c|}
\hline Estadístico & Valor \\
\hline Máximo & 56 \\
\hline Mínimo & 18 \\
\hline Media & 26,829 \\
\hline $\begin{array}{c}\text { Desviación } \\
\text { típica }\end{array}$ & 8,402 \\
\hline
\end{tabular}

Tabla 1. Edad de la muestra

En cuanto a la pregunta de si les gustan los ordenadores, un porcentaje mayoritario respondió afirmativamente (74,4\%). Y ninguno que no, afirmando una persona que le gustan para jugar (1,2\%) y el porcentaje restante $(24,4 \%)$ que le gustan un poco (Figura 6).

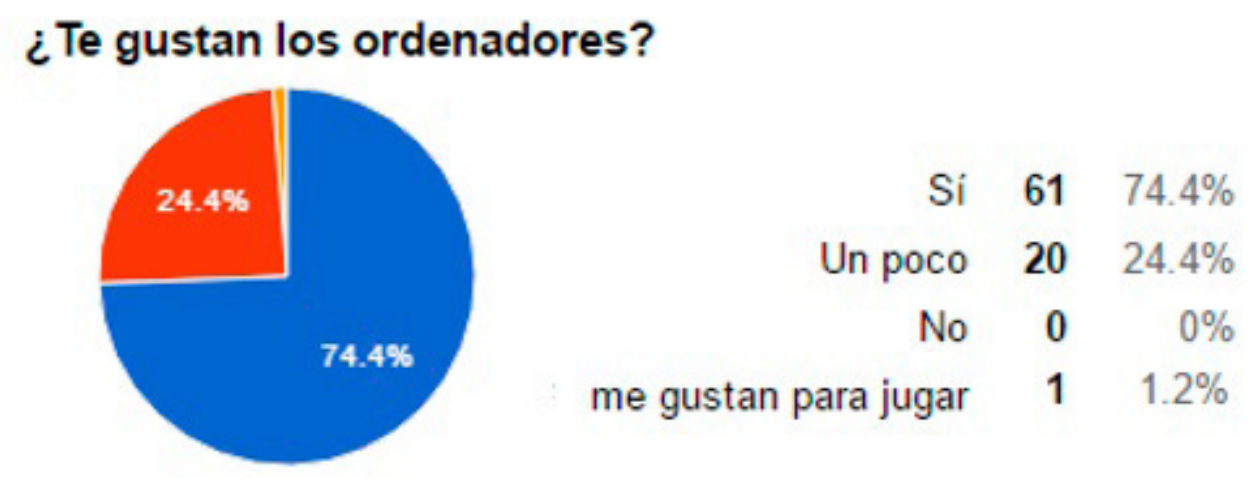

Figura 6. Gusto ordenadores

En cuanto a la disponibilidad de ordenador en su casa, el 100\% respondieron que sí (Figura 7). Y al acceso a internet en casa un porcentaje mayoritario $(97,8 \%)$ respondió que sí y el resto que no (Figura 8).

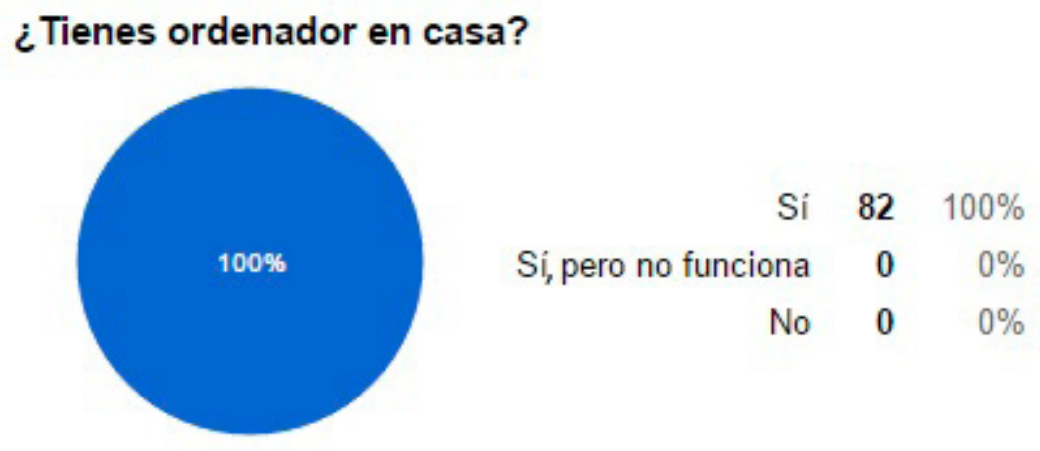




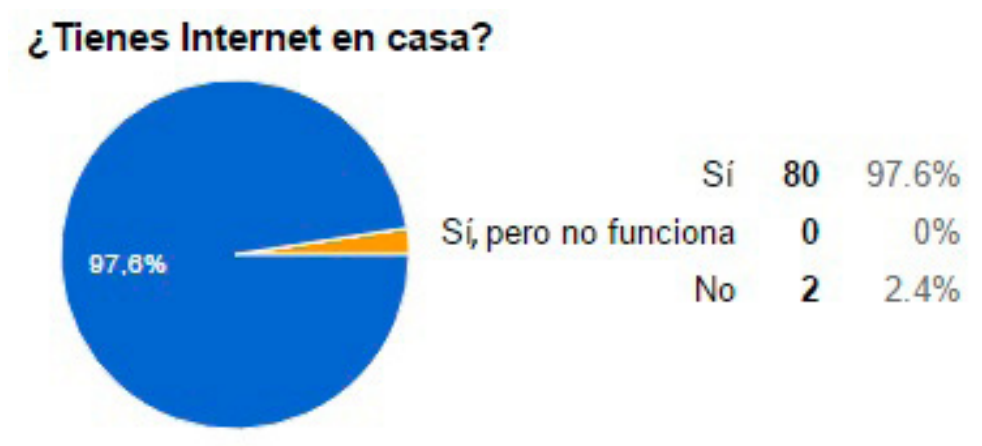

Figura 8. Tener Internet en casa

Respecto a la cuestión acerca de si tienen cuenta de correo, casi todos disponían de ella (97,6\%), excepto dos profesores que indicaron que no tenían, pero que les gustaría crearse una cuenta (Figura 9).

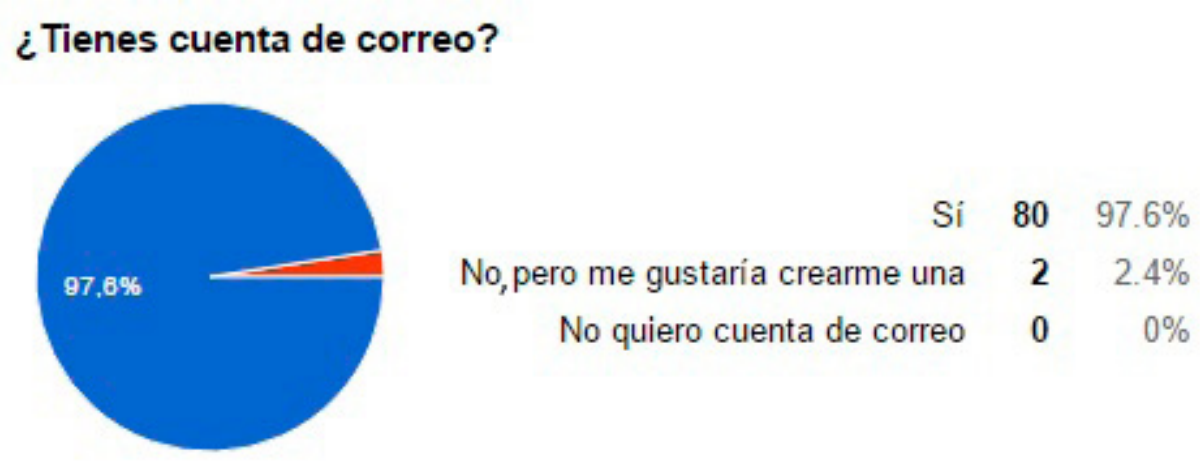

Figura 9. Tener cuenta de correo

En relación al uso de Internet para la búsqueda de recursos para llevar al aula y documentarse, como refleja la Figura 10 , el $43,9 \%$ indican que lo usan a diario, un $47,6 \%$ a veces y un $8,5 \%$ no lo usa.

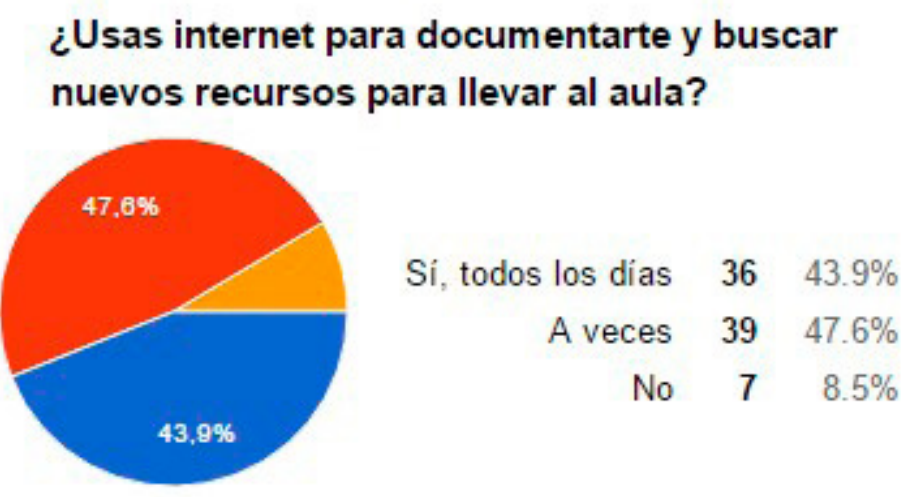

Figura 10. Uso de Internet

En cuanto al uso del ordenador para su trabajo, un porcentaje mayoritario hace uso de él, en casa y/o en el trabajo. Siendo tan solo un porcentaje ligeramente superior del $22 \%$ quienes no lo usan (Figura 11). 


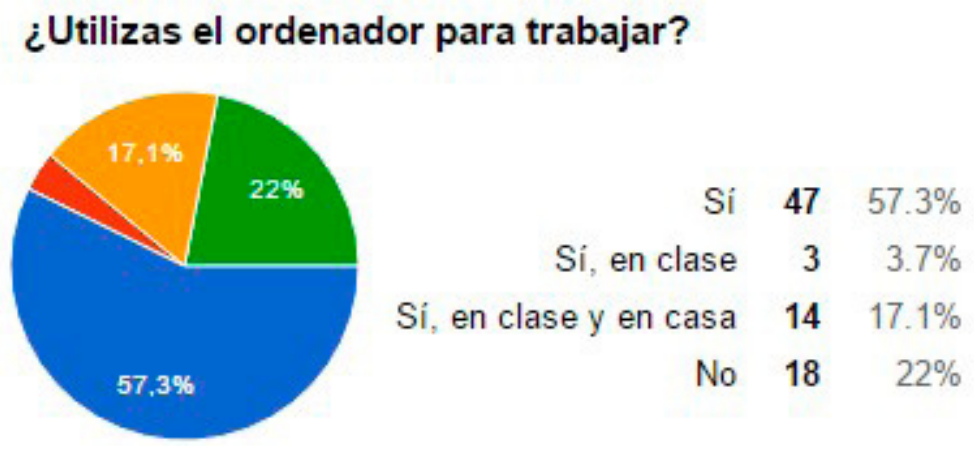

Figura 11. Uso de ordenador

En cuanto a las preguntas de valoración de 0 a 10, siendo 0 el menos importante, acerca de en caso de tener un programa en el ordenador que ayudara a sus estudiantes a repasar, cómo les gustaría que fuese o la importancia que daría diferentes aspectos, indicaron que fuera simpático, la importancia que se le dio a este aspecto fue bastante alta, como puede apreciarse en el histograma (Figura 12 y Tabla 2), valorando casi un 75\% de los docentes con 8, 9 o 10.

Si pudieras tener un programa en el ordenador que ayudara a tus estudiantes a repasar, puntúa de 0 (menos importante) a 10 (importancia máxima) la importancia que darías a que el programa fuera simpático:

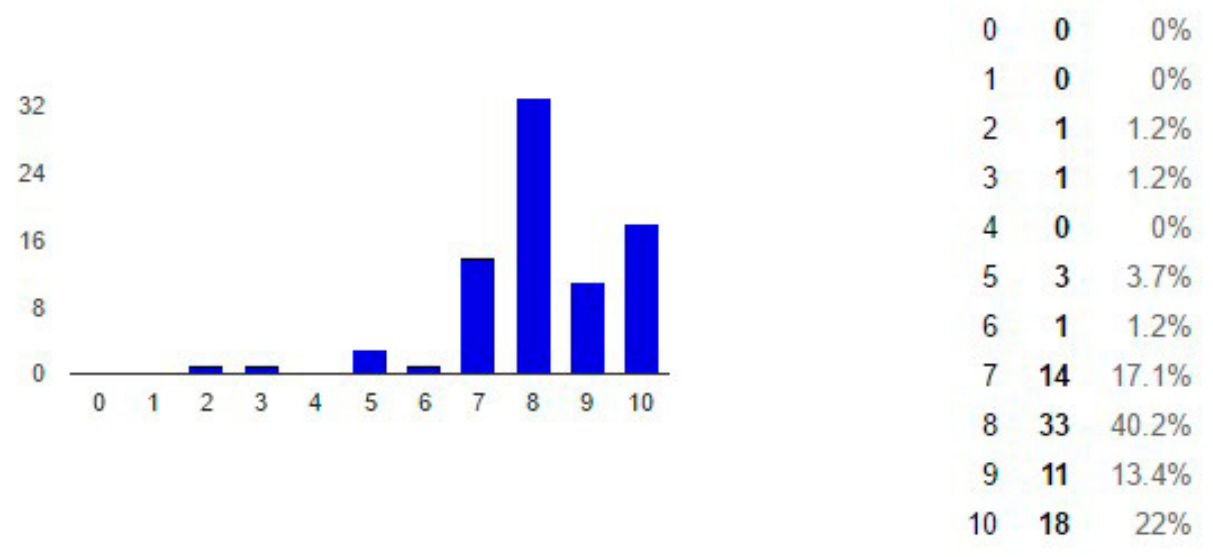

Figura 12. Ser simpático

\begin{tabular}{|l|l|}
\hline Estadístico & Valor \\
\hline Máximo & 10 \\
\hline Mínimo & 2 \\
\hline Media & 8,134 \\
\hline Desviación típica & 1,522 \\
\hline
\end{tabular}

Tabla 2. Ser simpático

En cuanto a la importancia de gestos con la cara (Figura 13 y Tabla 3) y con el cuerpo (Figura 14 y Tabla 4), le dan menos importancia, situándose el porcentaje mayoritario en 6, 7 y 8. 
Si pudieras tener un programa en el ordenador que ayudara a tus estudiantes a repasar, puntúa de 0 (menos importante) a 10 (importancia máxima) la importancia que darías a que el programa haga gestos con la cara:

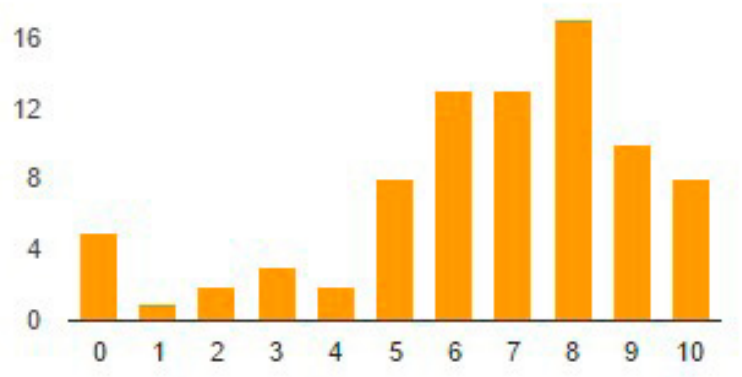

$\begin{array}{rrr}0 & \mathbf{5} & 6.1 \% \\ 1 & \mathbf{1} & 1.2 \% \\ 2 & \mathbf{2} & 2.4 \% \\ 3 & \mathbf{3} & 3.7 \% \\ 4 & \mathbf{2} & 2.4 \% \\ 5 & \mathbf{8} & 9.8 \% \\ 6 & \mathbf{1 3} & 15.9 \% \\ 7 & \mathbf{1 3} & 15.9 \% \\ \mathbf{8} & \mathbf{1 7} & 20.7 \% \\ 9 & \mathbf{1 0} & 12.2 \% \\ 10 & \mathbf{8} & 9.8 \%\end{array}$

Figura 13. Hace gestos con la cara

\begin{tabular}{|c|c|}
\hline Estadístico & Valor \\
\hline Máximo & 10 \\
\hline Mínimo & 0 \\
\hline Media & 6,549 \\
\hline Desviación típica & 2,616 \\
\hline
\end{tabular}

Tabla 3. Hacer gestos con la cara

Si pudieras tener un programa en el ordenador que ayudara a tus estudiantes repasar, puntúa de 0 (menos importante) a 10 (importancia máxima) la importancia que darías a que el programa haga gestos con el cuerpo:

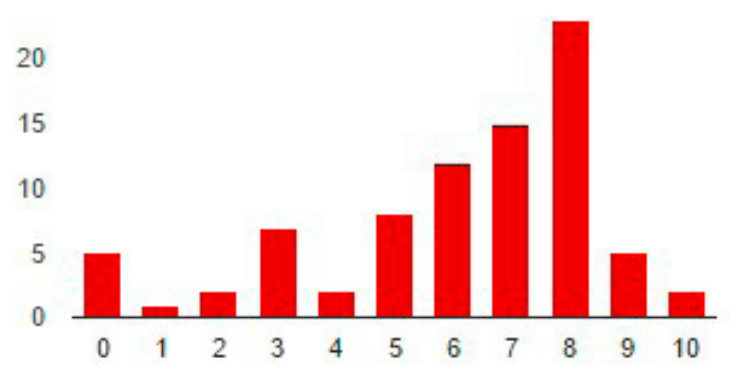

$\begin{array}{rrr}0 & \mathbf{5} & 6.1 \% \\ 1 & \mathbf{1} & 1.2 \% \\ 2 & \mathbf{2} & 2.4 \% \\ 3 & \mathbf{7} & 8.5 \% \\ 4 & \mathbf{2} & 2.4 \% \\ 5 & \mathbf{8} & 9.8 \% \\ 6 & \mathbf{1 2} & 14.6 \% \\ 7 & \mathbf{1 5} & 18.3 \% \\ 8 & \mathbf{2 3} & 28 \% \\ 9 & \mathbf{5} & 6.1 \% \\ 10 & \mathbf{2} & 2.4 \%\end{array}$

Figura 14. Hacer gestos con el cuerpo

\begin{tabular}{|l|l|}
\hline Estadístico & Valor \\
\hline Máximo & 10 \\
\hline Mínimo & 0 \\
\hline Media & 6,098 \\
\hline Desviación típica & 2,483 \\
\hline
\end{tabular}


Consideran bastante importante el hecho de que el programa dé consejos, valorando con 8 un 26,8\% con 9 un 24,4\% y con 20 un 28\% (Figura 15 y Tabla 5).

Si pudieras tener un programa en el ordenador que ayudara a tus estudiantes a repasar, puntúa de 0 (menos importante) a 10 (importancia máxima) la importancia que darías a que el programa te dé consejos:

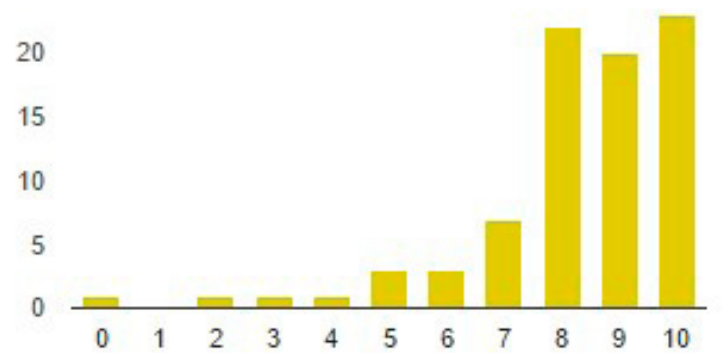

$\begin{array}{rrr}0 & \mathbf{1} & 1.2 \% \\ 1 & \mathbf{0} & 0 \% \\ 2 & \mathbf{1} & 1.2 \% \\ 3 & \mathbf{1} & 1.2 \% \\ 4 & \mathbf{1} & 1.2 \% \\ 5 & \mathbf{3} & 3.7 \% \\ 6 & \mathbf{3} & 3.7 \% \\ 7 & \mathbf{7} & 8.5 \% \\ 8 & \mathbf{2 2} & 26.8 \% \\ 9 & \mathbf{2 0} & 24.4 \% \\ 10 & \mathbf{2 3} & 28 \%\end{array}$

Figura 15. Dar consejos

\begin{tabular}{|l|l|}
\hline Estadístico & Valor \\
\hline Máximo & 10 \\
\hline Mínimo & 0 \\
\hline Media & 8,246 \\
\hline Desviación típica & 1,904 \\
\hline
\end{tabular}

Tabla 5. Dar consejos

La interacción en voz alta también la consideran importante (Figura 16 y Tabla 6), valorando un porcentaje superior al 75\% por encima de 6.

Si pudieras tener un programa en el ordenador que ayudara a tus estudiantes a repasar, puntúa de 0 (menos importante) a 10 (importancia máxima) la importancia que darías a que el programa te hable en voz alta:

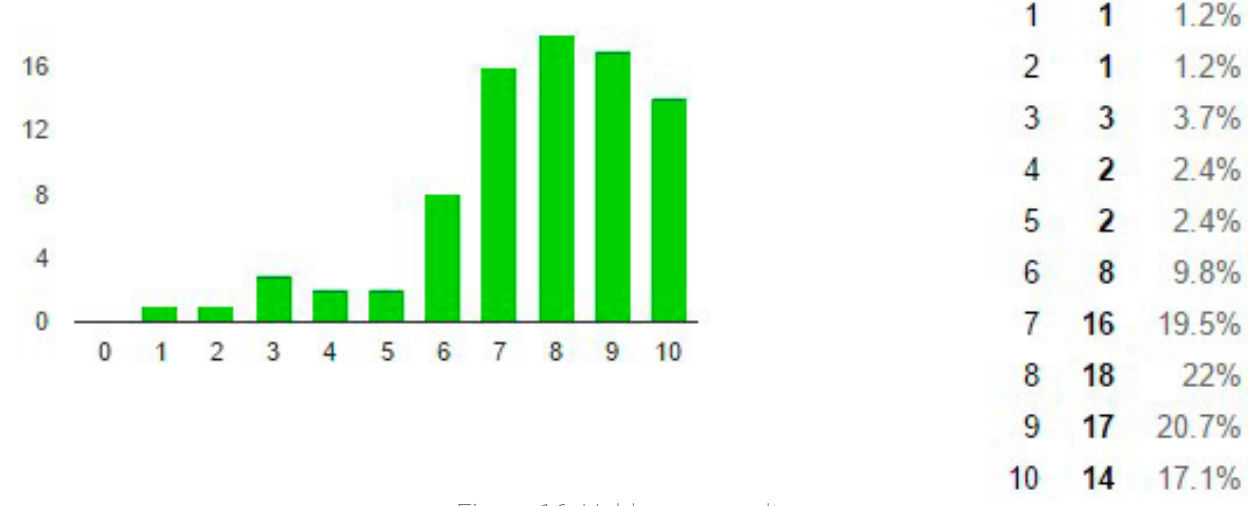

Figura 16. Hablar en voz alta 


\begin{tabular}{|l|l|}
\hline Estadístico & Valor \\
\hline Máximo & 10 \\
\hline Mínimo & 1 \\
\hline Media & 7,646 \\
\hline Desviación típica & 1,99 \\
\hline
\end{tabular}

Tabla 6. Hablar en voz alta

En relación a los aspectos de animar a seguir estudiando (Figura 17 y Tabla 7), que avise si te has equivocado (Figura 18 y Tabla 8), y que te recuerde tus opciones previas (Figura 19 y Tabla 9) los consideran de vital importancia, valorando más de un $80 \%$ con 7 o superior.

Si pudieras tener un programa en el ordenador que ayudara a tus estudiantes a repasar, puntúa de 0 (menos importante) a 10 (importancia máxima) la importancia que darías a que el programa te anime a seguir estudiando

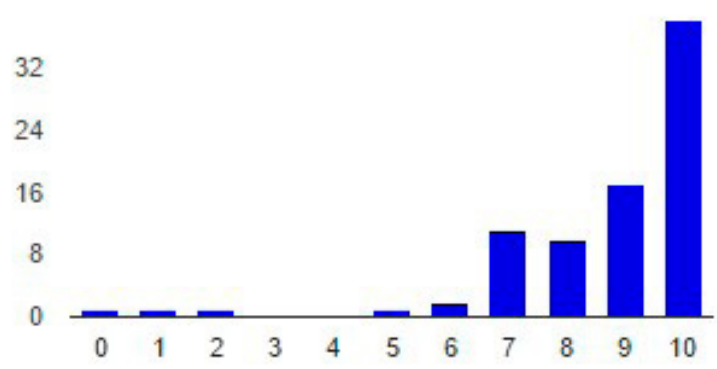

$\begin{array}{rrr}0 & \mathbf{1} & 1.2 \% \\ 1 & \mathbf{1} & 1.2 \% \\ 2 & \mathbf{1} & 1.2 \% \\ 3 & \mathbf{0} & 0 \% \\ 4 & \mathbf{0} & 0 \% \\ 5 & \mathbf{1} & 1.2 \% \\ 6 & \mathbf{2} & 2.4 \% \\ 7 & \mathbf{1 1} & 13.4 \% \\ 8 & \mathbf{1 0} & 12.2 \% \\ 9 & \mathbf{1 7} & 20.7 \% \\ 10 & \mathbf{3 8} & 46.3 \%\end{array}$

Figura 17. Animar a seguir estudiando

\begin{tabular}{|l|l|}
\hline Estadístico & Valor \\
\hline Máximo & 10 \\
\hline Mínimo & 0 \\
\hline Media & 8,659 \\
\hline Desviación típica & 1,958 \\
\hline
\end{tabular}

Tabla 7. Animar a seguir estudiando

Si pudieras tener un programa en el ordenador que ayudara a tus estudiantes a repasar, puntúa de 0 (menos importante) a 10 (importancia máxima) la importancia que darías a que el programa te diga en qué te has equivocado:

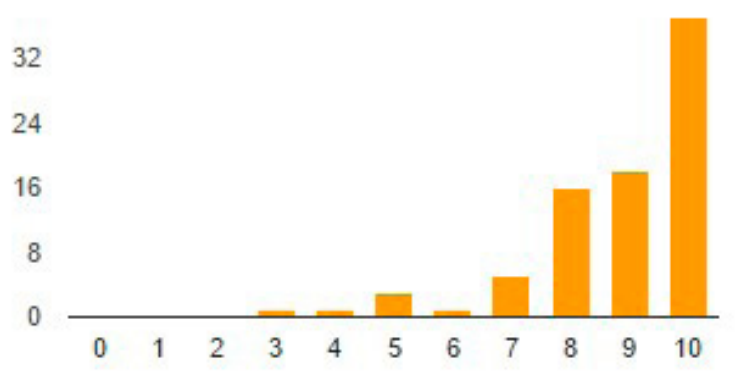

$\begin{array}{rrr}0 & 0 & 0 \% \\ 1 & 0 & 0 \% \\ 2 & 0 & 0 \% \\ 3 & \mathbf{1} & 1.2 \% \\ 4 & \mathbf{1} & 1.2 \% \\ 5 & 3 & 3.7 \% \\ 6 & \mathbf{1} & 1.2 \% \\ 7 & 5 & 6.1 \% \\ 8 & \mathbf{1 6} & 19.5 \% \\ 9 & \mathbf{1 8} & 22 \% \\ 10 & 37 & 45.1 \%\end{array}$




\begin{tabular}{|l|l|}
\hline Estadístico & Valor \\
\hline Máximo & 10 \\
\hline Mínimo & 3 \\
\hline Media & 8,817 \\
\hline Desviación típica & 1,525 \\
\hline
\end{tabular}

Tabla 8. Indicar equivocación

Si pudieras tener un programa en el ordenador que ayudara a tus estudiantes a repasar, puntúa de $\mathbf{0}$ (menos importante) a 10 (importancia máxima) la importancia que darías a que el programa recordase tusopciones previas:

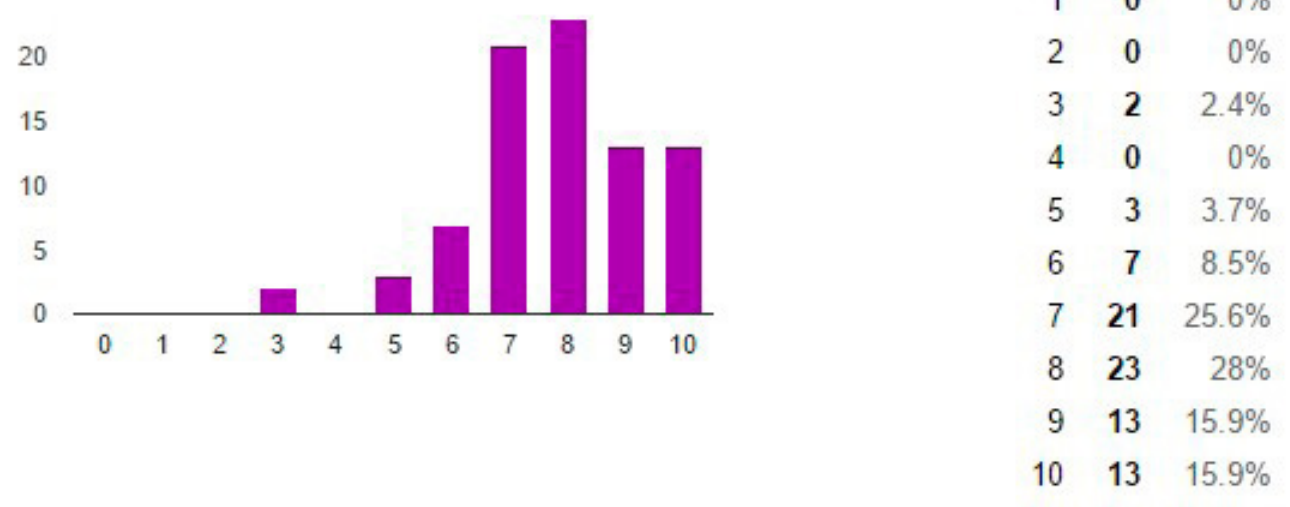

Figura 19. Recordar opciones previas

\begin{tabular}{|l|l|}
\hline Estadístico & Valor \\
\hline Máximo & 10 \\
\hline Mínimo & 3 \\
\hline Media & 7,817 \\
\hline Desviación típica & 1,525 \\
\hline
\end{tabular}

Tabla 9. Recordar opciones previas

Por último, consideran menos importante el hecho de que el programa tenga forma de ser humano

(Figura 20 y Tabla 10), habiendo valorado 50 personas (caso un 61\%) con valor 5 o inferior.

Si pudieras tener un programa en el ordenador que ayudara a tus estudiantes a repasar, puntúa de 0 (menos importante) a 10 (importancia máxima) la importancia que darías a que el programa tenga forma de ser humano:

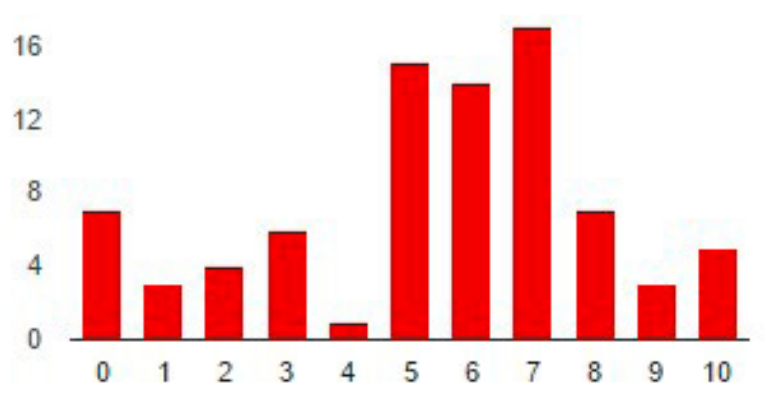

$\begin{array}{rrr}0 & 7 & 8.5 \% \\ 1 & 3 & 3.7 \% \\ 2 & 4 & 4.9 \% \\ 3 & 6 & 7.3 \% \\ 4 & \mathbf{1} & 1.2 \% \\ 5 & \mathbf{1 5} & 18.3 \% \\ 6 & 14 & 17.1 \% \\ 7 & \mathbf{1 7} & 20.7 \% \\ 8 & 7 & 8.5 \% \\ 9 & 3 & 3.7 \% \\ 10 & 5 & 6.1 \%\end{array}$




\begin{tabular}{|l|l|}
\hline Estadístico & Valor \\
\hline Máximo & 10 \\
\hline Mínimo & 0 \\
\hline Media & 5,415 \\
\hline Desviación típica & 2,694 \\
\hline
\end{tabular}

Tabla 10. Tener forma de ser humano

En cuanto a la opinión acerca si un sistema que ayudar a repasar puede motivar en el estudio (Figura 21), la respuesta mayoritaria con un $91,5 \%$ es que sí.

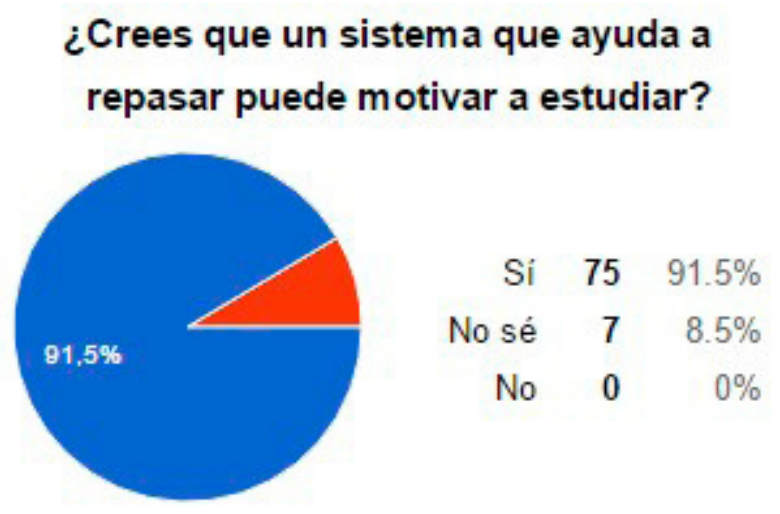

Figura 21. Motivar para estudiar

Se les pregunta sobre cómo creen que debería comportarse el programa si el alumno no está atento, a lo que responden 21 docentes que debería mostrar que si no estudia va a suspender, 16 le dicen que tiene que estudiar más, 5 que no haga nada, 4 que va a enviar un informe a los profesores, y la opción preferida es llamar la atención del estudiante con animación (36 docentes), los porcentajes correspondientes se pueden ver en la Figura 22.

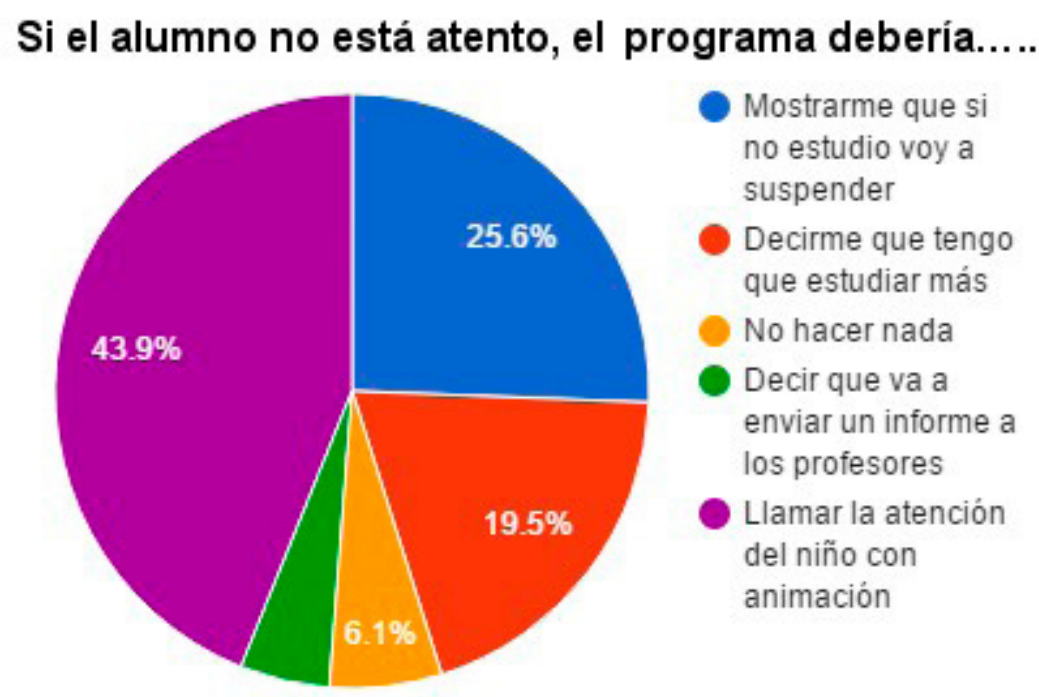

Figura 22. Alumno no atento 
Se realizan cuatro preguntas abiertas:

- Si el alumno no entiende algo, “¿qué te gustaría que hiciera el programa?”. La respuesta más repetida fue explicarlo de otra manera y apoyarse en material complementario como pueden ser ejemplos. Las respuestas más destacadas son las siguientes:

» Explicación de forma diferente (divertida, con otro vocabulario, sencilla, con juegos, gráficos, videos, etc.).

» Inclusión de ayuda, incitarle a que pregunte al profesor para resolver sus dudas.

¿ Ejemplos.

» Ampliación de información, recordatorio de contenidos.

» Guiar al alumno.

$»$ Tutorial.

» Que el agente pregunte qué es lo que el estudiante no comprende, motivarle mediante juegos para que intente coger el hilo de la explicación con el objetivo final de aprender.

» Explicar por qué son fallos y la reflexión sobre las soluciones.

» Animarle para que siga intentándolo.

- ¿Cuántas veces te gustaría usar el programa a la semana? ¿Por qué? Las respuestas van desde ninguno a todos los días, destacando la cifra de 1, 2, 3 y 4 veces. Las apreciaciones añadidas a las mismas han sido las siguientes:

» Un uso excesivo del ordenador en niños no les parece una idea correcta, es solo un apoyo.

» Dos profesores consideran que les gustaría para iniciar a los alumnos en el mundo de las TIC poco a poco.

» Todos los días después de clase para poder memorizar mejor los conocimientos y refrescarlos, repasar.

» No daría el protagonismo a tal programa, ya que considero que la interacción social y la interacción con elementos como el papel o témperas son mucho más productivos, sanos y divertidos.

» Dos veces por semana es suficiente, un uso mayor puede suponer que lo confundan con tiempo de ocio o distracción. 
»Es conveniente repasar todos los días lo que se da en clase ese mismo día.

¿ Es un complemento para el estudio.

» Un par de veces a la semana para que no acostumbren en exceso y porque el profesor siempre tiene que ser la referencia.

» Sí, para trabajar conceptos en los que los niños muestran más dificultad, es decir, dependiendo de lo que se trabaje esa semana lo usaría.

» Como un juego, es muy importante que sea divertido.

- ¿Te gustaría que los estudiantes pudiesen usar el programa en casa? ¿prefieres que lo usen en casa y/o en clase? La opción predominante es en ambos, destacando los siguientes comentarios:

» La supervisión por el profesor o los padres.

»El uso en casa de manera individual y en clase de forma colectiva y menos frecuente, ya que la figura del profesor es la que debe primar en clase.

»En ambos, si solo se usa en clase no le podrías sacar todo el provecho y en casa cada uno se puede administrar el tiempo para usarlo.

» Únicamente en clase bajo la supervisión responsable de alguien experto y que en casa se dedique a jugar y divertirse alejado de los aparatos electrónicos.

» En clase y así cuentan con el apoyo de sus compañeros y así fomentar el trabajo cooperativo.

» En casa, ofreciendo una ayuda más individualizada.

¿ En clase, ya que no todos los niños disponen de ordenador en casa.

»En los dos sitios, aunque más en casa, ya que en clase está el profesor y puede solucionar las dudas

- En general, ¿crees que el uso de este programa te puede ser útil? prácticamente todos estuvieron de acuerdo en que sí sería útil, aunque las respuestas fueron muy variadas. Muchos contestaron que serviría de apoyo en las explicaciones, otros señalaban que sería interesante poder trabajar con el agente desde casa y la importancia de integrar las TIC en la vida de los alumnos. Destacan las siguientes respuestas:

» Sí, es una forma de hacer el repaso más atractivo a los alumnos, ya que por lo general les encantan los ordenadores. Cualquier herramienta que ayude al estudio y a la motivación del alumno siempre es útil. 
Sí, porque además de las actividades de repaso que se hagan en el aula, los niños aprenden mejor con un medio audiovisual y este programa sería perfecto para repasar y motivarles a estudiar.

» Puede. Todo método educativo debe ser probado para ver los resultados en los alumnos.

» En un futuro estaría dispuesta a probarlo y ver si funciona.

» Puede ser útil a la hora de estudiar, ayudando a sacar mejores resultados académicos.

» Sí, ayudaría a afianzar lo visto en clase y a entenderlo de mejor manera.

» Puede llegar a ser muy útil e incluso podría ser indispensable, ya que se convertiría en una herramienta de uso diaria para muchos estudiantes y un gran apoyo.

»Por supuesto, nos ayudaría a ser constantes, terminar antes la tarea y a evitar frustraciones.

» Sí, porque te ayuda a repasar y reforzar los conocimientos y a comprenderlos mejor, lo cual a su vez no supondrá el abandono de los conocimientos que el profesor ha impartido en el aula tras finalizar la clase, sino que estos seguirán presentes en los alumnos.

» Sí, el repaso asienta el aprendizaje.

» Sí, puede ser muy útil en cuanto a motivación y técnicas de estudio.

» Sí, aprenden de forma lúdica y entretenida, lo cual tiene gran importancia a la hora del aprendizaje ya que influye en la motivación.

\section{Conclusiones}

Del estudio realizado puede concluirse para cada pregunta planteada al inicio lo siguiente:

P1) ¿Los profesores conocen esta tecnología educativa?

En general no. A la mayoría de los docentes, aunque estaban habituados al uso de Internet para la búsqueda de recursos para llevar al aula y documentarse (solo un 8,5\% no lo usa), y un 78\% usa el ordenador para trabajar, hubo que enseñarles una o varias imágenes de ACP para que supieran a qué se referían las preguntas de la encuesta.

P2) Si es así, ¿están acostumbrados al uso de este tipo de tecnologías en el aula?

Puesto que no conocían los ACP, no integraban esta tecnología en el aula. Cuando se les enseña, el $100 \%$ de los docentes encuestados consideran que es un buen sistema que ayuda a repasar y puede 
motivar al alumnado. Destacan que el número de veces que le gustaría usar el programa en clase sería entre 1-4 veces a la semana. Cuando se les ha preguntado si les gustaría que los estudiantes usasen el agente no solo en clase, sino también en casa, en algunos casos responden también afirmativamente.

P3) Si tuvieran poder de decisión en el diseño del agente, ¿qué características consideran que son las más adecuadas? ¿Cómo debería reaccionar el agente antes diversas situaciones?

Los aspectos que han considerado más relevantes son: si el alumno no entiende algo, es que el agente lo explicase de otra manera y apoyarse en material complementario como pueden ser ejemplos. En caso de que el alumno no esté atento, llamar su atención con animación.

En cuanto a los aspectos que debería incorporar el programa, los más destacados han sido que el programa diga que te has equivocado (media de 8,817 y desviación típica de 1,525), que anime a seguir estudiando (media de 8,659 y desviación típica de 1,958), que el programa proporcione consejos (con una media de 8,346 y desviación típica de 1,904), y que sea simpático (media de 8,134 y desviación típica de 1,522).

No se han encontrado agentes en la literatura que cumplan con todas estas características. En su lugar, hay agentes que las cumplen de forma parcial: AutoTutor, por ejemplo, tiene animación para mantener la atención, y los agentes de SBEL son sonrientes e intentan ser simpáticos para agradar en el aprendizaje del idioma.

La opinión de los docentes hacia el uso de las tecnologías en el aula y como parte del proceso de enseñanza de los profesores y aprendizaje de los niños es favorable, así como de los ACP (una vez que todos supieron lo que eran) es favorable.

Como trabajo futuro, se plantea ampliar la encuesta al mayor número de profesores posible, y seguir recabando más información para proporcionar más recomendaciones a diseñadores e investigadores en el área, y desarrollar así agentes que cumplan todas las características demandadas por los profesores.

También se tiene intención de realizar un análisis de correlaciones entre las respuestas recibidas por los profesores, para obtener más información, por ejemplo entre el perfil de los profesores y el tipo de agente que prefieren.

\section{Referencias}

Baylor, A., y Ebbers, S. (2003). Evidence that Multiple Agents Facilitate Greater Learning. International Artificial Intelligence in Education (AI-ED) Conference, Sydney, Australia. 
Biswas, G., Roscoe, R., Jeong, H., y Sulcer, B. (2009). Promoting self-regulated learning skills in agentbased learning environments. In Proceedings of the 17th international conference on computers in education (pp. 67-74).

Chase, C., Chin, D., Oppezzo, M., y Schwartz, D. (2009). Teachable agents and the protégé effect: Increasing the effort towards learning. Journal of Science Education and Technology, 18, 334-337. doi:https://doi.org/10.1007/s10956-009-9180-4

Clark, A., Fox, C., y Lappin, S. (Eds.). (2013). The handbook of computational linguistics and natural language processing. West Sussex, UK: John Wiley \& Sons.

D’mello, S., \& Graesser, A. (2012). AutoTutor and affective AutoTutor: Learning by talking with cognitively and emotionally intelligent computers that talk back. ACM Transactions on Interactive Intelligent Systems (TiiS), 2(4), 23. doi:https://doi.org/10.1145/2395123.2395128

Domínguez, J. (2011). Diseño de un asistente virtual. Tesis Fin de Carrera. Departamento de Sistemas de Computación. Instituto Tecnológico CD. Madero, Tamaulipas.

Graesser, A., Person, N., y Harter, D. (2001). Teaching tactics and dialog in AutoTutor. International Journal of Artificial Intelligence in Education, 12(3), 23-29.

Hays, M., Lane, C., Auerbach, D., Core, M., Gomboc, D., y Rosenberg, M. (2009). Feedback Specificity and the Learning of Intercultural Communication Skills, AIED.

Johnson, W., Rickel, J., y Lester, J. (2000). Animated Pedagogical Agents: Face-to-Face Interaction in Interactive Learning Environments. Journal of Artificial Intelligence in Education, 11, 47-78.

Khosrow-Pour, M. (Ed.). (2008). Encyclopedia of information science and technology (Vol. 1). Hershey PA, USA: IGI Global.

Kim, Y., y Baylor, A. (2006). A social cognitive framework for designing pedagogical agents as learning companions. Educational Technology Research and Development, 54(6), 569-596. doi:https://doi. org/10.1007/s11423-006-0637-3

Kuz, A., y Falco, M. (2015). Agent SocialMetric: herramienta de asistencia al docente para determinar el clima social y la estructura del aula. IE Comunicaciones: Revista Iberoamericana de Informática Educativa, 22, 16-29.

Leelawong, K., y Biswas, G. (2008). Designing Learning by Teaching Systems: The Betty's Brain System. International Journal of Artificial Intelligence in Education, 18(3), 181-208. 
Lester, J., Converse, S., Kahler, S., Barlow, S., Stone, B. y Bhogal, R. (1997). The persona effect: Affective impact of animated pedagogical agents. SIGCHI conference on Human factors in computing systems. Mas, A. (Ed.). (2005). Agentes software y sistemas multiagente: Conceptos, arquitecturas y aplicaciones. Madrid, España: Prentice Hall.

Morales-Rodríguez, M., y Domínguez-Martínez, J. (2011). Agentes Conversacionales como un Sistema de Diálogo. Memorias del V Encuentro de Investigadores del ITCM.

Nanne, M. F. (2015). Classification Criteria for Pedagogical Agents. International Journal of Computer Science and Technology, 6(3), 192-195.

Paulus, T. M., Horvitz, B., y Shi, M. (2006). 'Isn't it just like our situation?' Engagement and learning in an online story-based environment. Educational Technology Research and Development, 54(4), 355-385. doi:https://doi.org/10.1007/s11423-006-9604-2

Pérez-Marín, D. (2011). Uso de agentes conversacionales pedagógicos en sistemas de aprendizaje híbrido (b-learning). Actas del IV Seminario de Investigación en Tecnologías de la Información: SITIAE 2010 (pp. 79-94).

Pérez-Marín, D., y Pascual-Nieto, I. (2011). Conversational Agents and Natural Language Interaction: Techniques and Effective Practices. Hershey PA, USA: IGI Global. doi:https://doi.org/10.4018/978-160960-617-6

Person, N. K., y Graesser, A. C. (2000). Designing AutoTutor to be an effective conversational partner. In B. Fishman \& S. O'Connor-Divelbiss (Eds.), Fourth International Conference of the Learning Sciences (pp. 246-253). Mahwah, NJ: Erlbaum.

Reategui, E. B., y Moraes, M. C. (2006). Agentes pedagógicos animados. Novas Tecnologias na Educação, 4(2), 1-10.

Reategui, E., Polonia, E., y Roland, L. (2007). The role of animated pedagogical agents in scenario-based language e-learning: a case-study. In Conference ICL2007, September 26-28, 2007. Kassel University Press.

Ryu, J., y Baylor, A. (2005). The Psychometric Structure of Pedagogical Agent Persona. Technology, Instruction, Cognition \& Learning, 2, 291-314.

Ryokai, K., Vaucelle, C., y Cassell, J. (2003). Virtual peers as partners in storytelling and literacy learning. Journal of Computer Assisted Learning, 19(2), 195-208. doi: https://doi.org/10.1046/j.02664909.2003.00020.x 
Schroeder, N. L., Adesope, O. O., y Gilbert, R. B. (2013). How effective are pedagogical agents for learning? A meta-analytic review. Journal of Educational Computing Research, 49(1), 1-39. doi:https:// doi.org/10.2190/EC.49.1.a

Smith, T., Affleck, G., Lees, B., y Branki, C. (1999). Implementing a generic framework for a web-based pedagogical agent. In Annual Australassian Society for Computers in Learning in Tertiary Education Conference, Brisbane, Queesland.

Tamayo-Moreno, S. (2012). Propuesta de Desarrollo Centrado en el Usuario de un Agente Conversacional Pedagógico para la Comprensión Lectora de Ejercicios de Matemáticas a nivel escolar. Trabajo Fin de Máster. Máster Universitario en Investigación en Sistemas Hardware y Software Avanzados de la Escuela Técnica Superior de Ingeniería Informática de la Universidad Rey Juan Carlos.

Tamayo-Moreno, S. (2017). Propuesta de Metodología para el Diseño e Integración en el Aula de un Agente Conversacional Pedagógico desde Educación Secundaria hasta Educación Infantil. Tesis Doctoral. Universidad Rey Juan Carlos, Móstoles, Madrid, España.

Tatar, D., Sacarea, C., y Kapetanios, E. (2013). Natural Language Processing: Semantic Aspects. Boca Raton, FL: CRC Press.

VanLehn, K., Graesser, A. C., Jackson, G. T., Jordan, P., Olney, A., y Rosé, C. P. (2007). When are tutorial dialogues more effective than reading? Cognitive Science, 31(1), 3-62. doi:https://doi. org/10.1080/03640210709336984

Van Vuuren, S. (2007). Technologies that empower pedagogical agents and visions for the future. Educational Technology, 47(1), 4-10.

Veletsianos, G., Miller, C., y Doerin, A. (2009). Enali: A Research and Design Framework for Virtual Characters and Pedagogical Agents. Journal of Educational Computing Research, 41(2), 171-194. doi:https://doi.org/10.2190/EC.41.2.c

Yee, N., y Bailenson, J. (2007). The Proteus effect: The effect of transformed self-representation on behavior. Human Communication Research, 33, 3. doi:https://doi.org/10.1111/j.14682958.2007.00299.x

Ziegahn, L. (2001). 'Talk' about culture online: The potential for transformation. Distance Education, 22(1), 144-150. doi:https://doi.org/10.1080/0158791010220109 